\title{
Article
}

\section{Pre-diagnostic plasma bile acid levels and colon cancer risk: A prospective study}

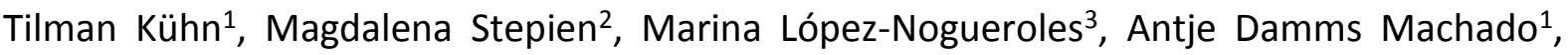
Disorn Sookthai ${ }^{1}$, Theron Johnson ${ }^{1}$, Marta Roca ${ }^{3}$, Anika Hüsing ${ }^{1}$, Sandra González Maldonado ${ }^{1}$, Amanda J. Cross ${ }^{4}$, Neil Murphy², Heinz Freisling ${ }^{5}$, Sabina Rinaldi ${ }^{6}$, Augustin Scalbert ${ }^{6}$, Veronika Fedirco ${ }^{7}$, Gianluca Severi ${ }^{8,9}$, Marie-Christine Boutron-Ruault ${ }^{8,9}$, Francesca Romana Mancini ${ }^{8,9}$, Solomon A. Sowah ${ }^{1}$, Heiner Boeing ${ }^{10}$, Paula Jakszyn ${ }^{11,12}$, Maria-Jose Sánchez ${ }^{13,14}$, Susana Merino ${ }^{15}$, Sandra Colorado-Yohar ${ }^{14,16,17}$, Aurelio Barricarte ${ }^{14,18,19}$, Kay Tee Khaw ${ }^{20}$; Julie A. Schmidt ${ }^{21}$, Aurora Perez-Cornago ${ }^{21}$, Antonia Trichopoulou ${ }^{22}$, Anna Karakatsani ${ }^{22,23}$, Paschalis Thriskos ${ }^{22}$, Domenico Palli2 ${ }^{24}$, Claudia Agnoli ${ }^{25}$, Rosario Tumino ${ }^{26}$, Carlotta Sacerdote ${ }^{27}$, Salvatore Panico ${ }^{28}$, Bas Bueno-de-Mesquita ${ }^{29,30,31,32}$, Carla H. van Gils ${ }^{33}$, Alicia Heath ${ }^{4}$, Marc J. Gunter ${ }^{2}$, Elio Riboli ${ }^{4}$ Agustín Lahoz ${ }^{3}$, Mazda Jenab², and Rudolf Kaaks ${ }^{1}$

Corresponding author: Dr Tilman Kühn, German Cancer Research Center, Division of Cancer Epidemiology, Im Neuenheimer Feld 280, D-69120 Heidelberg, Germany; Telephone: +49 622142 3184; Fax: +49 622142 2203; Email: t.kuehn@dkfz.de

${ }^{1}$ German Cancer Research Center (DKFZ), Division of Cancer Epidemiology, Heidelberg, Germany

${ }^{2}$ International Agency for Research on Cancer (IARC), Nutritional Epidemiology Group, Section of Nutrition and Metabolism, Lyon, France

${ }^{3}$ Health Research Institute Hospital La Fe, Analytical Unit, Biomarkers and Precision Medicine Unit Valencia, Spain

(C) The Author(s) 2019. Published by Oxford University Press. All rights reserved. For permissions, please email: journals.permissions@oup.com 
${ }^{4}$ School of Public Health, Imperial College London, St Mary's Campus, London, United Kingdom

${ }^{5}$ International Agency for Research on Cancer (IARC), Nutritional Methodology and

Biostatistics Group, Section of Nutrition and Metabolism, Lyon, France

${ }^{6}$ International Agency for Research on Cancer (IARC), Biomarkers Group, Section of Nutrition and Metabolism, Lyon, France

77Department of Epidemiology, Rollins School of Public Health, Winship Cancer Institute, Emory University, Atlanta, USA

${ }^{8}$ CESP, Fac. de médecine - Univ. Paris-Sud, Fac. de médecine - UVSQ, INSERM, Université Paris-Saclay, 94805, Villejuif, ${ }^{9}$ Gustave Roussy, F-94805, Villejuif, France

${ }^{10}$ Department of Epidemiology, German Institute of Human Nutrition Potsdam-Rehbruecke, Nuthetal, Germany

${ }^{11}$ Unit of Nutrition and Cancer, Cancer Epidemiology Research Programme, Catalan Institute of Oncology, L’Hospitallet de Llobregat, Barcelona, Spain

${ }^{12}$ Facultat de Ciències de la Salut Blanquerna, Universitat Ramón Llull, Barcelona, Spain

${ }^{13}$ Escuela Andaluza de Salud Pública. Instituto de Investigación Biosanitaria ibs.GRANADA, Universidad de Granada. Granada, Spain

${ }^{14}$ CIBER de Epidemiología y Salud Pública (CIBERESP), Madrid, Spain

${ }^{15}$ Public Health Directorate, Asturias, Spain

${ }^{16}$ Department of Epidemiology, Murcia Regional Health Council, IMIB-Arrixaca, Murcia, Spain

${ }^{17}$ Research Group on Demography and Health, National Faculty of Public Health, University of Antioquia, Medellín, Colombia

${ }^{18}$ Navarra Public Health Institute, Pamplona, Spain 
${ }^{19}$ Navarra Institute for Health Research (IdiSNA) Pamplona, Spain

${ }^{20}$ University of Cambridge School of Clinical Medicine, Clinical Gerontology Unit, Addenbrooke's Hospital, Cambridge, United Kingdom

${ }^{21}$ Cancer Epidemiology Unit, Nuffield Department of Population Health, University of Oxford, Oxford, United Kingdom

${ }^{22}$ Hellenic Health Foundation, Athens, Greece

${ }^{23}$ 2nd Pulmonary Medicine Department, School of Medicine, National and Kapodistrian University of Athens, "ATTIKON" University Hospital, Haidari, Greece

${ }^{24}$ Cancer Risk Factors and Life-Style Epidemiology Unit, Institute for Cancer Research, Prevention and Clinical Network - ISPRO, Florence, Italy

${ }^{25}$ Epidemiology and Prevention Unit, Fondazione IRCCS Istituto Nazionale dei Tumori, Milan, Italy

${ }^{26}$ Cancer Registry and Histopathology Department, "Civic - M.P. Arezzo" Hospital, ASP Ragusa, Italy

${ }^{27}$ Unit of Cancer Epidemiology, Città della Salute e della Scienza University-Hospital and Center for Cancer Prevention (CPO), Turin, Italy

${ }^{28}$ Dipartimento di Medicina Clinica e Chirurgia Federico II University, Naples, Italy ${ }^{29}$ Former senior scientist, Dept. for Determinants of Chronic Diseases (DCD), National Institute for Public Health and the Environment (RIVM), Bilthoven, The Netherlands ${ }^{30}$ Former associate professor, Department of Gastroenterology and Hepatology, University Medical Centre, Utrecht, the Netherlands

${ }^{31}$ Former visiting professor, School of Public Health, Imperial College London, St Mary's Campus, London, United Kingdom 
${ }^{32}$ Former Academic Icon / visiting professor, Dept. of Social \& Preventive Medicine, Faculty of Medicine, University of Malaya, Kuala Lumpur, Malaysia

33Julius Centre for Health Sciences and Primary Care, University Medical Centre Utrecht, Utrecht, the Netherlands

Abbreviations: CA (Cholic Acid); CDCA (Chenodeoxycholic Acid); $\mathrm{Cl}$ (Confidence Interval); DCA (Deoxycholic Acid); FXR (Farnesoid X receptor); GCA (Glycocholic Acid); GCDCA (Glycochenodeoxycholic Acid); GDCA (Glycodeoxycholic Acid); GHCA (Glycohyocholic Acid); GLCA (Glycolithocholic Acid); GUDCA (Glycoursodeoxycholic Acid); HCA (Hyocholic Acid); ICD (International Classification of Diseases); ICD-O (International Classification of Diseases for Oncology); LOD (Limit of Detection); LCA (lithocholic acid); MRM (multiple reaction monitoring); MS (mass spectrometry); OR (Odds Ratio); TaMCA (Tauro-Alpha-Muricholic Acid); TCA (Taurocholic Acid); TCDCA (Taurochenodeoxycholic Acid); TDCA (Taurodeoxycholic Acid); THCA (Taurohyocholic Acid); TUDCA (Tauroursodeoxycholic Acid); UDCA (Ursodeoxycholic Acid); UPLC (ultraperformance liquid chromatography); 


\section{Abstract}

Background: Bile acids have been proposed to promote colon carcinogenesis. However, there are limited prospective data on circulating bile acid levels and colon cancer risk in humans.

Methods: Associations between pre-diagnostic plasma levels of 17 primary, secondary and tertiary bile acid metabolites (conjugated and unconjugated) and colon cancer risk were evaluated in a nested case-control study within the European Prospective Investigation into Cancer and Nutrition (EPIC) cohort. Bile acid levels were quantified by tandem mass spectrometry in samples from 569 incident colon cancer cases and 569 matched controls. Multivariable logistic regression analyses were used to estimate odds ratios (ORs) for colon cancer risk across quartiles of bile acid concentrations.

Results: Positive associations were observed between colon cancer risk and plasma levels of 7 conjugated bile acid metabolites, i.e. primary bile acids glycocholic acid (ORQuartile 4 vs. Quartile ${ }_{1}=2.22,95 \%$ confidence interval $\left.[\mathrm{Cl}]=1.52,3.26\right)$, taurocholic acid $(\mathrm{OR}=1.78,95 \% \mathrm{Cl}=1.23$, 2.58), glycochenodeoxycholic acid $(\mathrm{OR}=1.68,95 \% \mathrm{Cl}=1.13,2.48)$, taurochenodeoxycholic acid $(\mathrm{OR}=1.62,95 \% \mathrm{Cl}=1.11-2.36)$, and glycohyocholic acid $(\mathrm{OR}=1.65,95 \% \mathrm{Cl}=1.13,2.40)$ as well as the secondary bile acids glycodeoxycholic acid (OR=1.68, 95\%Cl=1.12, 2.54) and taurodeoxycholic acid $(\mathrm{OR}=1.54,95 \% \mathrm{Cl}=1.02,2.31)$. By contrast, unconjugated bile acids and tertiary bile acids were not associated with risk.

Conclusions: This prospective study showed that pre-diagnostic levels of certain conjugated primary and secondary bile acids were positively associated with risk of colon cancer. Our findings support experimental data to suggest that a high bile acid load is colon cancer promotive. 
Bile acids constitute a group of endogenous cholesterol-derived metabolites with detergent properties. Primary bile acids cholic acid (CA) and chenodeoxycholic acid (CDCA) are synthesized in the liver and largely conjugated to glycine or taurine. Conjugation increases their water solubility, lowers their toxicity, and is a precondition for the storage of bile in the gallbladder. Bile acids are released into the small intestine in response to a cholecystokinin stimulus upon meal ingestion. In the gut lumen, bile acids facilitate transport, digestion and absorption of nutrients, particularly lipids and lipid-soluble vitamins. Up to $95 \%$ of bile acids are actively re-absorbed in the terminal ileum and transported back to the liver. Only a small fraction of bile acids escape this efficient enterohepatic circulation and enter the systemic circulation. Importantly, bile acids are subject to transformations by the intestinal microbiota, which deconjugate them, and, to a lesser extent dehydroxylize them into the free secondary bile acids deoxycholic acid (DCA, derived from CA) and lithocholic acid (LCA, derived from CDCA). In addition, CDCA is partially transformed into the tertiary bile acid ursodeoxycholic acid (UDCA) by gut bacteria [1-4].

It has long been proposed that bile acids, particularly secondary bile acids, may promote colon carcinogenesis by inducing DNA and protein damage as well as mucosal cell proliferation and inflammation $[3,5,6]$. More recently, impaired function of the Farnesoid X receptor (FXR), a nuclear bile acid receptor that regulates bile acid homeostasis, has also been shown to facilitate colon cancer development [4]. However, some evidence suggests beneficial roles of bile acids [7]. For example, the tertiary bile acid UDCA, used in the treatment of cholangitis, may be anti-carcinogenic according to laboratory-based mechanistic studies [8]. Yet, UDCA supplementation trials among patients with ulcerative colitis and primary sclerosing cholangitis have shown mixed results with regard to colon cancer risk [8]. 
Epidemiological studies on fecal bile acid composition and colon cancer risk have been inconsistent. The only bile acid that was statistically significantly increased in the feces of colorectal adenoma and cancer patients in a meta-analysis of small case-control studies was CDCA, while levels of secondary bile acids were not elevated [9]. Heterogeneity in results from the included studies may well be related to large variation in fecal bile acid content and difficulties with sampling standardization [5]. Thus, it has been suggested that blood levels of bile acids could be more useful to investigate associations with cancer risk than fecal bile acids, and small case-control studies have found increased serum DCA levels in patients with colon cancer [10-12]. As prospective studies on bile acid profiles and colon cancer risk are currently lacking, the aim of the present study was to evaluate, for the first time, the associations between pre-diagnostic plasma bile acid concentrations and colon cancer risk in the European Prospective Investigation into Cancer and Nutrition (EPIC), using a validated targeted approach for bile acid quantification [13].

\section{Methods}

\section{Study Population}

EPIC is an ongoing multicenter cohort study carried out across 23 study sites in 10 Western-European countries. The study was designed to investigate associations between nutritional, metabolic, hormonal, lifestyle as well as genetic factors and cancer risk, and a detailed study protocol has previously been published [14]. In short, 521,448 adults ( 70\% female) aged 35 to 75 years were recruited for EPIC between 1992 and 2000. At baseline, detailed questionnaire- and interview-derived information on habitual diet, lifestyle, 
reproductive factors, and health status was obtained, and anthropometric measurements were taken. Blood was drawn following standardized procedures, and processed into serum, plasma, buffy coat, and erythrocyte samples. The majority of these samples were stored at $-196^{\circ} \mathrm{C}$ in liquid nitrogen at the International Agency for Research on Cancer (IARC, Lyon, France). Samples from the Danish and Swedish centers were stored locally at $-150^{\circ}$ in nitrogen vapor and $-80^{\circ} \mathrm{C}$ in freezers, respectively. For financial and logistical reasons, samples of participants from Denmark, Sweden, and Norway were not included in the present analyses.

Since baseline, incident cases of cancer are ascertained by linkage to cancer registries (Denmark, Sweden, Norway, Italy, Spain, the Netherlands, and the United Kingdom), or by health insurance records, cancer and pathology registries, and active follow-up with subsequent validation of potential cases by clinical records (Naples, France, Germany, and Greece). The International Classification of Diseases (ICD-10) and the International Classification of Diseases for Oncology (ICD-O-2) were used to identify and classify incident cases of colon cancer (C18).

Cases of primary colon cancer diagnosed between 1993 and $2008(n=581)$ were included in the current nested case-control study. Each case was matched to a control subject free of cancer (with the exception of non-melanoma skin cancer) at the index date, i.e. the date of diagnosis of the cancer case. An incidence density sampling protocol with age ( \pm 6 months at recruitment), sex, study center, follow-up time, time of day at blood collection ( $\pm 4 \mathrm{~h}$ ), fasting status (less than 3 hours, 3-6 hours, and more than 6 hours), menopausal status (premenopausal, perimenopausal, postmenopausal, or surgically menopausal), current use of exogenous hormones (oral contraceptives or hormone therapy, 
yes/no), and phase of menstrual cycle at blood collection as the underlying matching criteria was used.

The present study was approved by the ethics committees of the IARC and the individual study centers. All participants provided written informed consent.

\section{Laboratory Methods}

Bile acid concentrations were measured at the Analytical Unit of the Health Research Institute Hospital La Fe (Valencia, Spain) using a validated and published ultraperformance liquid chromatography/multiple reaction monitoring/mass spectrometry (UPLC-MRM-MS) method [13]. Briefly, $50 \mu \mathrm{L}$ of plasma was spiked with deuterated internal standards stock solution. Following this, proteins were precipitated and samples were dried and reconstituted in $50 \mu \mathrm{L}$ methanol:water (50:50, V/V). Samples were analyzed using an Acquity UPLC system (Waters, UK) equipped with an Acquity UPLC BEH C18 column $(1.7 \mu \mathrm{m}$, $2.1 \times 100 \mathrm{~mm}$; Waters). The MS analysis was performed using a Waters Xevo TQ-S mass spectrometer (Waters) with an ESI source working in the negative-ionization mode. This targeted profiling method was initially developed to quantify 31 bile acid metabolites in different matrices, of which 17 are detectable in human blood.

Prior to the present analyses on bile acids and colon cancer, we showed that the biological reproducibility of most bile acid metabolites was good (with Spearman's coefficients $>0.5$ for intra-individual correlations over one year, see Supplementary Methods and Supplementary Table 1). 
The laboratory personnel were blinded to the case-control status of the samples throughout the entire measurement series. For logistical reasons samples were divided into 6 different batches for injection. Intra- and inter-batch precision were calculated using an extra sample from a healthy donor, treated exactly as the other samples and injected repeatedly. All coefficients of variation were below 15\%. An overview of the bile acid metabolite levels as well as the number of values below the limit of detection (LOD) is given in Supplementary Table 2. The bile acid with the highest proportion of concentrations below the LOD was taurohyocholic acid (THCA), with $54.0 \%$ missing values among cases and $50.3 \%$ missing values among controls, followed by tauroursodeoxycholic acid (TUDCA, 28.6\%/27.1\%), tauro-alpha-muricholic acid (TaMCA, 18.3\%/14.1\%), and ursodeoxycholic acid (UDCA, $15.3 \% / 14.8 \%$ ), while all other bile acids were detectable in more than $95 \%$ of the samples. For statistical analyses, values below the LOD were assigned half the LOD.

\section{Statistical Analyses}

Spearman's rank coefficients were calculated to evaluate correlations between bile acid metabolites among controls. Generalized Linear Models were used to evaluate associations between bile acid levels and the covariates used for logistic regression analyses listed below (Supplementary Methods). Conditional logistic regression models were used to obtain odds ratios (ORs) and 95\% confidence intervals (Cls) for colon cancer across quartiles of individual bile acid concentrations. Analyses were conditioned on the matching factors (age, sex, study center, follow-up time, time of day at blood collection, fasting status, menopausal status, current use of exogenous hormones, and phase of menstrual cycle), and further adjusted for education level (no school degree or primary school; secondary school; technical or professional school; longer education / university degree), physical activity level 
(active, moderately active, moderately inactive, inactive; according to the Cambridge Index [15]), smoking status (never, former, current), waist circumference $(\mathrm{cm})$, height $(\mathrm{cm})$, and consumption of red and processed meat (g/day), fat (g/day), fiber (g/day), and energy (kcal/day). Linear trends were assessed fitting bile acid concentrations continuously on the log2 scale as independent variables, as tests for non-linearity by restricted cubic splines did not indicate non-linear associations.

Multiplicative interaction between covariates and bile acid levels was tested for including cross-product terms in the logistic regression models, and heterogeneity in associations between bile acids and colon cancer risk by sex and by anatomical site (proximal vs. distal) was assessed by $X^{2}$ tests. Sensitivity analyses were carried out excluding cases that had occurred during the first two years of follow-up from logistic regression analyses. We further corrected odds ratios from logistic regression using product-moment correlation coefficients [16] from our above-mentioned reproducibility study (Supplementary Methods). Finally, we tested whether concentrations of individual bile acids relative to the total of all metabolite concentrations were associated with colon cancer. Statistical tests were all two-sided, and differences were considered as statistically significant at $p$-values $<0.05$. SAS 9.4 (Cary, NC, USA) was used for the present analyses. 


\section{Results}

\section{Study Population}

Plasma volumes of 12 of the selected 581 case-control pairs were not sufficient for bile acid measurements so the final sample consisted of 569 case-control pairs. The median age of cases and controls at blood collection was 57.5 years (Table 1). The median follow-up duration was 6.0 years (range: $0.1-14.4$ years). Overall, $62.6 \%$ of the participants were female. The majority of samples (65.0\%) were taken after a fasting duration of at least 6 hours, $16.0 \%$ after 3 to 6 hours, and $19.0 \%$ after up to 3 hours. There was a tendency for higher waist circumference values and lower self-reported physical activity among cases compared to controls, while other established risk factors for colorectal cancer showed similar median values and distributions. Median bile acid concentrations tended to be higher among cases, particularly for primary and secondary bile acids (Table 2).

Spearman's rank coefficients $(\rho)$ for correlations between bile acid metabolites are shown in Supplementary Figure 1. High correlations $(\rho>0.7)$ were observed between unconjugated primary bile acids ( $C A, C D C A$, and $\mathrm{HCA}$ ), between conjugated primary bile acids (GCA, TCA, TCDCA and GCDCA), and between conjugated secondary bile acids (GDCA and TDCA). Bile acid concentrations were higher among men (Supplementary Table 3), and lower with longer fasting duration (Supplementary Table 4). While several metabolites were further associated with alcohol consumption (either inversely, or with higher levels among both non-consumers and high consumers), associations with other covariates were sporadic (Supplementary Table 5 - 9). 


\section{Bile Acids and Colon Cancer Risk}

Associations between concentrations of individual bile acids and colon cancer risk are shown in Figure 1, and in more detail in Table 3. We observed statistically significant positive associations between most of the conjugated primary bile acids (GCA, TCA, GCDCA, TCDCA, and GHCA) and colon cancer risk when comparing extreme quartiles (Figure 1 and Table 3). The strongest association was between GCA and colon cancer risk (OR quartile 4 vs. quartile 1: 2.22 [95 \% Cl: 1.52, 3.26], plinear trend=0.002), followed by TCA $(1.78[1.23,2.58])$, $\operatorname{GCDCA}(1.68[1.13,2.48]), \operatorname{GHCA}(1.65[1.13,2.40])$, and $\operatorname{TCDCA}(1.62[1.11,2.36])$. By contrast, none of the unconjugated primary bile acids (CA, CDCA, HCA) showed a statistically significant association with colon cancer. Among the secondary bile acids, the unconjugated metabolite DCA was not associated with colon cancer, while our analyses showed statistically significant positive associations for the conjugated metabolites GDCA and TDCA, with ORs [95\% Cls] of $1.68[1.12,2.54]$ and 1.54 [1.02, 2.31], respectively (Figure 1 and Table 3). None of the tertiary bile acids were associated with colon cancer (Figure 1 and Table 3). Upon correction of $p$-values for multiple testing by the Bonferroni method (multiplying $p$ values by the number of bile acid metabolites), only the association between GCA and colon cancer remained statistically significant ( $p_{\text {linear }}$ trend $=0.032$ ). Similarly, only GCA was statistically significantly associated with colon cancer risk in a mutually adjusted model including the other bile acid metabolites that were associated with colon cancer in the above-mentioned multivariable models.

Analyses on THCA and colon cancer risk by quartiles were not possible, as more than $50 \%$ of THCA values were below the LOD. Modelling THCA levels as a dichotomous variable (values below the LOD vs. detectable values) or a continuous trend variable on the log2 
scale did not show associations with colon cancer. A similar pattern of associations between all other bile acid metabolites with colon cancer was found when values below the LOD where excluded instead of assigning values at half the LOD. ORs only marginally changed when cases that had occurred during the first two years of follow-up were excluded from the analyses, and stratifying logistic regression analyses by median follow-up duration showed no statistically significant heterogeneity (data not shown).

We did not observe associations between total primary, total secondary, total conjugated, and total unconjugated bile acids, or their ratios with colon cancer risk. The ratio between DCA and CA, for which a positive association with colon cancer was shown in a smaller study among British women [10], was not associated with colon cancer risk in our study, neither overall nor in analyses stratified by sex (data not shown). When using relative rather than absolute bile acid concentrations in logistic regression models, only GCA showed a statistically significant association with colon cancer (OR: $1.48[1.04,2.12], p_{\text {linear trend }}=0.09$, see Supplementary Table 10). There were no statistically significant interactions between bile acids and possible effect modifiers in relation to colon cancer risk, and no statistically significant heterogeneity by anatomic site was observed (Supplementary Table 11). Although statistical analyses did not show heterogeneity by sex across associations between bile acid metabolites and colon cancer, most associations between individual bile acids, particularly conjugated primary bile acids, and colon cancer risk tended to be stronger among women than among men (Figure 2 and Supplementary Table 12).

Upon correction of associations between bile acids and colon cancer for measurement error, some of the ORs substantially increased (Supplementary Table 13). 
Most strikingly, the OR for colon cancer among participants in the highest quartile of GCA concentrations was $2.22[1.52,3.26]$ before and $4.78[2.01,29.89]$ after correction.

\section{Discussion}

In this large prospective study, we investigated whether pre-diagnostic concentrations of circulating bile acids were related to the risk of colon cancer. We observed that plasma levels of five conjugated primary bile acids (GCA, TCA, GCDCA, TCDCA and GHCA) as well as two conjugated secondary bile acids (GDCA, TDCA) were statistically significantly associated with increased colon cancer risk. Overall, these findings are supportive of a large body of prior experimental evidence, and in accordance with smaller epidemiological studies, to suggest that higher bile acid load may promote colon cancer.

Most previous epidemiological studies on bile acids and colorectal cancer risk were case-control comparisons of fecal bile acid contents. These studies showed that concentrations of CDCA (unconjugated primary bile acid) and LCA as well as DCA (unconjugated secondary bile acids) tended to be higher in samples of colorectal cancer patients than in samples of controls, although results were inconsistent [9]. With regard to circulating bile acids, two small case-control studies from the 1990 s indicated that concentrations of DCA (particularly the unconjugated fraction), were higher in sera of patients with colorectal adenomas compared to controls [11, 12]. A smaller prospective study of women from the United Kingdom (46 incident cases and matched controls from a cohort of 6127 women, with a mean age of 55 years at baseline) showed no statistically 
significant associations between major bile acid metabolites and colorectal cancer, even though there was a tendency for increased risk with a higher DCA / CA ratio [10].

The above-mentioned findings from smaller epidemiological studies are consistent with experimental data to indicate colon cancer promoting effects of unconjugated secondary bile acids via DNA- and protein damage, increased genomic instability, apoptosis resistance, and enhanced cell proliferation in the colonic epithelium $[17,18]$. Our observation that conjugated rather than unconjugated primary and secondary bile acids in the circulation were associated with colon cancer may be due to the fact that bacterial deconjugation and de-hydroxylation, which increases the concentrations of cancer promoting unconjugated secondary bile acids (DCA, LCA), mostly occurs in the large intestine, whereas bile acids undergoing the enterohepatic cycle are reabsorbed in the terminal ileum, i.e. prior to bacterial transformations [4]. In line with this notion, proportions of unconjugated secondary bile acids are much higher in feces than in the circulation of healthy individuals, even if unconjugated secondary bile acids may occur in the systemic circulation due to passive absorption in the large intestine [19].

Another interesting line of evidence on bile acids and colon cancer risk comes from a comprehensive prospective metabolomics study among 254 cases and 254 controls embedded in the Prostate, Lung, Colorectal, and Ovarian Cancer Screening Trial (PLCO) [20]. In this study, out of 676 serum metabolites, GCDCA (conjugated primary) was most strongly associated with colorectal cancer risk among female study participants (OR: 5.34) [20]. Further strong positive associations were observed for GCA and TCDCA (conjugated primary) and TDCA (conjugated secondary) among women. These results are in agreement with ours of stronger associations between concentrations of GCA, TCDCA, and TDCA and colon 
cancer among women, although we did not observe formal statistical heterogeneity by sex for any of the bile acids. Unlike in our study, the tertiary bile acid GUDCA was associated with colon cancer risk among women in the PLCO. Reasons for the described potential sex differences remain unclear and further studies are needed to investigate possible underlying mechanisms.

Our study was the first large prospective study with a targeted quantification of prediagnostic blood levels of bile acids in relation to colon cancer risk. Overall, it supports the hypothesis of an increased bile acid load promoting colon carcinogenesis. However, despite the large case number, our sample size may have been limited for some of the presented subgroup analyses with smaller strata. We did not have the opportunity to compare blood levels of bile acids with fecal levels in our study (although issues related to the standardization of native fecal samples may speak against such comparisons) and data on the composition of the intestinal microbiome are not available in EPIC. Moreover, we could not detect LCA, the most cytotoxic secondary bile acid, in its unconjugated form in most of our samples, although our assay allows a detection of LCA levels between 0.02 to $0.04 \mu \mathrm{M}$ as reported from previous studies [10, 21]. Given that blood bile acids including LCA seem to be rather stable with respect to storage conditions and sample processing [10, 22], we have no obvious explanation for the lack of LCA values in our analyses. Our reproducibility study suggests that a single assessment of plasma bile acid concentrations may be sufficient for prospective studies, although the risk associations we observed may have been underestimated due to regression dilution. Only a few metabolites, particularly UDCA, showed lower biological reproducibility, which may explain the null associations with colon cancer risk for UDCA in our study. Considering experimental evidence to suggest that bile acid concentrations can be modulated by dietary interventions or drugs [23, 24], increased 
bile acids could be modifiable and amenable to prevention and treatment. As we could not assess potential interventions to manipulate bile acid concentrations, future studies are needed to better understand the effects of specific dietary or medical interventions on the concentrations of individual bile acids in blood and feces in humans.

In summary, we observed positive associations between pre-diagnostic plasma concentrations of bile acids, particularly conjugated primary and secondary bile acids, and colon cancer. Our data support experimental evidence to indicate that a higher bile acid load constitutes a risk factor for colon cancer. While it has been suggested that bile acid concentrations can be manipulated by dietary modification and drugs, future studies are needed on the effects of specific interventions on bile acid concentrations in human blood and feces.

\section{Funding}

Bile acid measurements in the present study were funded by Helmholtz Association of German Research Centers via the project Aging and Metabolic Programming (AMPro, DKFZ). The present study was further co-financed by the European Union through the Operational Program of the European Regional Development Fund (ERDF) of the Valencian Community 2014-2020 (Hospital La Fe), and by the European Regional Development Fund (FEDER) Institute of Health Carlos III of the Spanish Ministry of Economy and Competitiveness (PI17/01282).

The coordination of EPIC is financially supported by the European Commission (DGSANCO) and the International Agency for Research on Cancer. The national cohorts are 
supported by the following funders: Ligue Contre le Cancer, Institut Gustave Roussy, Mutuelle Générale de l'Education Nationale, Institut National de la Santé et de la Recherche Médicale (INSERM) (France); German Cancer Aid, German Cancer Research Center (DKFZ), Federal Ministry of Education and Research (BMBF) (Germany); the Hellenic Health Foundation (Greece); Associazione Italiana per la Ricerca sul Cancro-AIRC-Italy and National Research Council (Italy); Dutch Ministry of Public Health,Welfare and Sports (VWS), Netherlands Cancer Registry (NKR), LK Research Funds, Dutch Prevention Funds, Dutch ZON (Zorg Onderzoek Nederland),World Cancer Research Fund (WCRF), Statistics Netherlands (The Netherlands); Health Research Fund (FIS, PI13/00061 to Granada, PI13/01162 to EPICMurcia, PI13/02633 to EPIC-Navarra), Regional Governments of Andalucía, Asturias, Basque Country, Murcia and Navarra, ISCIII RETIC (RD06/0020) (Spain); Cancer Research UK (14136 to EPIC-Norfolk; C570/A16491 and C8221/A19170 to EPIC-Oxford), Medical Research Council (1000143 to EPIC-Norfolk, MR/M012190/1 to EPIC-Oxford) (UK).

\section{Notes}

The study funders had no role in the design of the study; the collection, analysis, or interpretation of the data; the writing of the manuscript; or the decision to submit the manuscript for publication. The authors have no conflicts of interest to report.

Data and analyses are made available upon reasonable request. For information on how to submit an application for gaining access to EPIC data and/or biospecimens, please follow the instructions at: http://epic.iarc.fr/access/index.php.

Where authors are identified as personnel of the International Agency for Research on Cancer / World Health Organization, the authors alone are responsible for the views 
expressed in this article and they do not necessarily represent the decisions, policy or views of the International Agency for Research on Cancer / World Health Organization.

\section{References}

1. Kuipers F, Bloks VW, Groen AK. Beyond intestinal soap--bile acids in metabolic control. Nat Rev Endocrinol 2014;10(8):488-98.

2. Martinot $E$, Sedes L, Baptissart M, et al. Bile acids and their receptors. Mol Aspects Med 2017; 10.1016/j.mam.2017.01.006.

3. Rowland I, Gibson G, Heinken A, et al. Gut microbiota functions: metabolism of nutrients and other food components. Eur J Nutr 2017; 10.1007/s00394-017-1445-8.

4. Degirolamo C, Modica S, Palasciano G, et al. Bile acids and colon cancer: Solving the puzzle with nuclear receptors. Trends Mol Med 2011;17(10):564-72.

5. Costarelli V. Bile acids as possible human carcinogens: new tricks from an old dog. Int J Food Sci Nutr 2009;60 Suppl 6:116-25.

6. Renga B, Mencarelli A, Cipriani S, et al. Molecular determinants of gastrointestinal and liver cancers: role of bile acid activated nuclear receptors. Curr Top Med Chem 2012;12(6):625-36.

7. Phelan JP, Reen FJ, Caparros-Martin JA, et al. Rethinking the bile acid/gut microbiome axis in cancer. Oncotarget 2017;8(70):115736-115747.

8. Ocvirk S, O’Keefe SJ. Influence of Bile Acids on Colorectal Cancer Risk: Potential Mechanisms Mediated by Diet-Gut Microbiota Interactions. Current Nutrition Reports $2017 ; 6(4): 315-322$ 
9. Tong JL, Ran ZH, Shen J, et al. Association between fecal bile acids and colorectal cancer: a meta-analysis of observational studies. Yonsei Med J 2008;49(5):792-803.

10. Costarelli V, Key TJ, Appleby PN, et al. A prospective study of serum bile acid concentrations and colorectal cancer risk in post-menopausal women on the island of Guernsey. Br J Cancer 2002;86(11):1741-4.

11. Bayerdorffer E, Mannes GA, Richter WO, et al. Increased serum deoxycholic acid levels in men with colorectal adenomas. Gastroenterology 1993;104(1):145-51.

12. Bayerdorffer E, Mannes GA, Ochsenkuhn T, et al. Unconjugated secondary bile acids in the serum of patients with colorectal adenomas. Gut 1995;36(2):268-73.

13. Garcia-Canaveras JC, Donato MT, Castell JV, et al. Targeted profiling of circulating and hepatic bile acids in human, mouse, and rat using a UPLC-MRM-MS-validated method. J Lipid Res 2012;53(10):2231-41.

14. Riboli E, Kaaks R. The EPIC Project: rationale and study design. European Prospective Investigation into Cancer and Nutrition. Int J Epidemiol 1997;26 Suppl 1:S6-14.

15. Wareham NJ, Jakes RW, Rennie KL, et al. Validity and repeatability of a simple index derived from the short physical activity questionnaire used in the European Prospective Investigation into Cancer and Nutrition (EPIC) study. Public Health Nutr 2003;6(4):407-13.

16. Frost C, Thompson SG. Correcting for regression dilution bias: comparison of methods for a single predictor variable. JSTOR 2000;163(2):173-189.

17. Ocvirk S, O'Keefe SJD. Influence of Bile Acids on Colorectal Cancer Risk: Potential Mechanisms Mediated by Diet-Gut Microbiota Interactions. Current Nutrition Reports $2017 ; 6(4): 315-322$

18. Louis P, Hold GL, Flint HJ. The gut microbiota, bacterial metabolites and colorectal cancer. Nat Rev Microbiol 2014;12(10):661-72. 
19. Ridlon JM, Kang D-J, Hylemon PB. Bile salt biotransformations by human intestinal bacteria. 2006;47(2):241-259.

20. Cross AJ, Moore SC, Boca S, et al. A prospective study of serum metabolites and colorectal cancer risk. Cancer 2014;120(19):3049-57.

21. Frommherz L, Bub A, Hummel E, et al. Age-Related Changes of Plasma Bile Acid Concentrations in Healthy Adults--Results from the Cross-Sectional KarMeN Study. PLoS One 2016;11(4):e0153959.

22. Xiang $X$, Han $Y$, Neuvonen $M$, et al. High performance liquid chromatography-tandem mass spectrometry for the determination of bile acid concentrations in human plasma. J Chromatogr B Analyt Technol Biomed Life Sci 2010;878(1):51-60.

23. Ridlon JM, Kang DJ, Hylemon PB, et al. Bile acids and the gut microbiome. Curr Opin Gastroenterol 2014;30(3):332-8.

24. Schramm C. Bile Acids, the Microbiome, Immunity, and Liver Tumors. N Engl J Med 2018;379(9):888-890. 
Tables

Table 1 Baseline characteristics of the study population

\begin{tabular}{|c|c|c|}
\hline Characteristic & Controls ( $n=569)$ & Cases $(n=569)$ \\
\hline $\begin{array}{l}\text { Median age at blood collection, } y \\
(\min , \max )\end{array}$ & $57.5(36.7,74.3)$ & $57.5(36.7,74.3)$ \\
\hline \multicolumn{3}{|l|}{ Sex n (\%) } \\
\hline Females & $356(62.6)$ & $356(62.6)$ \\
\hline Males & $213(37.4)$ & $213(37.4)$ \\
\hline \multicolumn{3}{|l|}{ Menopausal status n (\%) } \\
\hline Premenopausal & $72(20.2)$ & $69(19.4)$ \\
\hline Postmenopausal & $240(67.4)$ & $238(66.9)$ \\
\hline Perimenopausal & $25(7.0)$ & $27(7.6)$ \\
\hline Surgical postmenopausal & $19(5.3)$ & $22(6.2)$ \\
\hline \multicolumn{3}{|l|}{$\begin{array}{l}\text { Current use of exogenous } \\
\text { hormones } \mathrm{n}(\%)\end{array}$} \\
\hline Yes & 46 (12.9) & $47(13.2)$ \\
\hline No & $309(86.8)$ & $307(86.2)$ \\
\hline Not specified & $1(0.3)$ & $2(0.6)$ \\
\hline \multicolumn{3}{|l|}{ Fasting status $\mathrm{n}(\%)$} \\
\hline $0-3$ hours & $108(19.0)$ & $108(19.0)$ \\
\hline$>3-6$ hours & $91(16.0)$ & $91(16.0)$ \\
\hline$>6$ hours & $370(65.0)$ & $370(65.0)$ \\
\hline $\begin{array}{l}\text { Median waist circumference, } \mathrm{cm} \\
\left(25^{\text {th }} \text { percentile, } 75^{\text {th }} \text { percentile }\right)\end{array}$ & $87.0(78.0,96.0)$ & $89.0(79.2,98.5)$ \\
\hline $\begin{array}{l}\text { Median height, } \mathrm{cm}\left(25^{\text {th }} \text { percentile, }\right. \\
\left.75^{\text {th }} \text { percentile }\right)\end{array}$ & $163.0(156.5,171.0)$ & $164.2(158.5,170.5)$ \\
\hline $\begin{array}{l}\text { Median acohol intake at } \\
\text { recruitment, } \mathrm{g} / \mathrm{d} \text { ( } 25^{\text {th }} \text { percentile, } \\
75^{\text {th }} \text { percentile) }\end{array}$ & $6.0(0.6,21)$ & $5.6(0.4,23.8)$ \\
\hline $\begin{array}{l}\text { Median red meat consumption, } \\
\mathrm{g} / \mathrm{d}\left(25^{\text {th }} \text { percentile, } 75^{\text {th }}\right. \\
\text { percentile) }\end{array}$ & $40.8(23.3,67.7)$ & $38.7(20.4,59.8)$ \\
\hline Median Processed meat & & \\
\hline $\begin{array}{l}\text { consumption, } \mathrm{g} / \mathrm{d}\left(25^{\text {th }} \text { percentile, }\right. \\
75^{\text {th }} \text { percentile) }\end{array}$ & $22.3(9.7,41.1)$ & $23.0(8.9,41.6)$ \\
\hline $\begin{array}{l}\text { Median Fiber consumption, } \mathrm{g} / \mathrm{d} \\
\left(25^{\text {th }} \text { percentile, } 75^{\text {th }} \text { percentile }\right)\end{array}$ & $21.9(17.8,26.8)$ & $21.7(17.5,26.5)$ \\
\hline \multicolumn{3}{|l|}{ Smoking status $\mathrm{n}(\%)$} \\
\hline Never & $295(51.8)$ & $269(47.4)$ \\
\hline Former & 159 (27.9) & $172(30.2)$ \\
\hline Current & $112(19.7)$ & $123(21.6)$ \\
\hline Unknown & $3(0.5)$ & $5(0.9)$ \\
\hline
\end{tabular}




\begin{tabular}{lll}
$\begin{array}{ll}\text { Education level n (\%) } \\
\text { Primary school / no school degree }\end{array}$ & $222(49.7)$ & $212(48.5)$ \\
$\begin{array}{l}\text { Secondary school (\%) } \\
\text { Technical / professional school }\end{array}$ & $81(14.2)$ & $106(18.6)$ \\
$\begin{array}{l}\text { Longer education / university } \\
\text { degree }\end{array}$ & $90(15.8)$ & $86(15.1)$ \\
Not specified & $10(1.8)$ & $90(15.8)$ \\
$\begin{array}{l}\text { Cambridge physical activity index n } \\
\text { (\%) }\end{array}$ & & $11(1.9)$ \\
Inactive & $167(29.3)$ & \\
Moderately inactive & $213(37.4)$ & $181(31.8)$ \\
Moderately active & $94(16.5)$ & $230(40.4)$ \\
Active & $92(16.2)$ & $91(16.0)$ \\
Missing & $3(0.5)$ & $65(11.4)$ \\
\hline
\end{tabular}




\begin{tabular}{|c|c|c|c|}
\hline Bile acid metabolite & Classification & $\begin{array}{c}\text { Controls } \\
\text { Median( } 25^{\text {th }} \text { percentile, } \\
\left.75^{\text {th }} \text { percentile }\right) \\
(n=569) \\
\end{array}$ & $\begin{array}{c}\text { Cases } \\
\text { Median( } 25^{\text {th }} \text { percentile, } \\
\left.75^{\text {th }} \text { percentile }\right) \\
(n=569)\end{array}$ \\
\hline Cholic Acid (CA) & Primary, unconjugated & $76.5(26.8,312.7)$ & $91.7(30.6,353.3)$ \\
\hline Chenodeoxycholic Acid (CDCA) & Primary, unconjugated & $150.4(55.9,432)$ & $164.3(61.4,455.6)$ \\
\hline Hyocholic Acid (HCA) & Primary, unconjugated & $7.2(3.0,17.4)$ & $7.4(2.9,15.7)$ \\
\hline Glycocholic Acid (GCA) & Primary, conjugated & $94.9(48.2,187.9)$ & $119.8(60.4,233.5)$ \\
\hline Taurocholic Acid (TCA) & Primary, conjugated & $12.9(5.3,27.4)$ & $15.0(6.4,37.4)$ \\
\hline $\begin{array}{l}\text { Glycochenodeoxycholic Acid } \\
\text { (GCDCA) }\end{array}$ & Primary, conjugated & $462.9(224.3,786.9)$ & $496.8(275.1,927.2)$ \\
\hline Taurochenodeoxycholic (TCDCA) & Primary, conjugated & $45.0(20.4,90.2)$ & $50.5(24.6,102.9)$ \\
\hline Glycohyocholic Acid (GHCA) & Primary, conjugated & $7.2(5.0,13.1)$ & $7.9(5.0,13.8)$ \\
\hline Taurohyocholic Acid (THCA) & Primary, conjugated & $1.2(1.2,2.5)$ & $1.2(1.2,2.5)$ \\
\hline $\begin{array}{l}\text { Tauro-Alpha-Muricholic Acid } \\
\text { (TaMCA) }\end{array}$ & Primary, conjugated & $3.4(2.5,6.8)$ & $3.8(2.5,7.1)$ \\
\hline Deoxycholic Acid (DCA) & Secondary, unconjugated & $217.5(99.1,427.8)$ & $239.3(113.3,433.8)$ \\
\hline Glycodeoxycholic Acid (GDCA) & Secondary, conjugated & $107.2(48.0,228.4)$ & $125.3(60.4,254.9)$ \\
\hline Taurodeoxycholic Acid (TDCA) & Secondary, conjugated & $14.5(6.1,34.0)$ & $17.0(7.8,39.6)$ \\
\hline Glycolithocholic Acid (GLCA) & Secondary, conjugated & $10.1(4.8,23.9)$ & $9.6(4.9,22.0)$ \\
\hline Ursodeoxycholic Acid (UDCA) & Tertiary, unconjugated & $23.1(9.1,55.1)$ & $22.9(8.4,56.1)$ \\
\hline Glycoursodeoxycholic Acid (GUDCA) & Tertiary, conjugated & $59.1(28.8,105.4)$ & $55.5(30.9,111.0)$ \\
\hline Tauroursodeoxycholic Acid (TUDCA) & Tertiary, conjugated & $2.5(1.2,4.6)$ & $2.5(1.2,5.0)$ \\
\hline
\end{tabular}


Table 3 Odds ratios (95\% confidence intervals) for colon cancer across quartiles of bile acids*

\begin{tabular}{|c|c|c|c|c|c|}
\hline Bile acid metabolite & Quartile 1 & Quartile 2 & Quartile 3 & Quartile 4 & $\mathbf{p}_{\text {trend }} \boldsymbol{t}^{\dagger}$ \\
\hline \multicolumn{6}{|c|}{ Primary unconjugated bile acids } \\
\hline \multicolumn{6}{|l|}{ Cholic Acid (CA) } \\
\hline N Cases (\%) & $127(22.3)$ & $140(24.6)$ & $147(25.8)$ & $155(27.2)$ & \\
\hline OR $(95 \% \mathrm{Cl})$ & Reference & $1.17(0.82,1.66)$ & $1.18(0.81,1.72)$ & $1.32(0.90,1.94)$ & 0.27 \\
\hline \multicolumn{6}{|c|}{ Chenodeoxycholic Acid (CDCA) } \\
\hline N Cases (\%) & $134(23.6)$ & $143(25.1)$ & $141(24.8)$ & $151(26.5)$ & \\
\hline OR $(95 \% \mathrm{Cl})$ & Reference & $1.01(0.70,1.45)$ & $1.0(0.69,1.45)$ & $1.12(0.77,1.64)$ & 0.76 \\
\hline \multicolumn{6}{|c|}{ Hyocholic Acid (HCA) } \\
\hline N Cases (\%) & $152(26.7)$ & $126(22.1)$ & $164(28.8)$ & $127(22.3)$ & \\
\hline OR $(95 \% \mathrm{Cl})$ & Reference & $0.84(0.58,1.20)$ & $1.11(0.78,1.58)$ & $0.89(0.61,1.31)$ & 0.60 \\
\hline \multicolumn{6}{|c|}{ Primary conjugated bile acids } \\
\hline \multicolumn{6}{|c|}{ Glycocholic Acid (GCA) } \\
\hline N Cases (\%) & $102(17.9)$ & $142(25.0)$ & $133(23.4)$ & $192(33.7)$ & \\
\hline OR $(95 \% \mathrm{Cl})$ & Reference & $1.46(1.00,2.12)$ & $1.47(0.99,2.19)$ & $2.22(1.52,3.26)$ & 0.002 \\
\hline \multicolumn{6}{|c|}{ Taurocholic Acid (TCA) } \\
\hline N Cases (\%) & $111(19.5)$ & $142(25.0)$ & $132(23.2)$ & $184(32.3)$ & \\
\hline OR $(95 \% \mathrm{Cl})$ & Reference & $1.25(0.87,1.81)$ & $1.23(0.84,1.79)$ & $1.78(1.23,2.58)$ & 0.01 \\
\hline \multicolumn{6}{|c|}{ Glycochenodeoxycholic Acid (GCDCA) } \\
\hline N Cases (\%) & $107(18.8)$ & $164(28.8)$ & $130(22.8)$ & $168(29.5)$ & \\
\hline OR $(95 \% \mathrm{Cl})$ & Reference & $1.46(1.03,2.05)$ & $1.19(0.81,1.76)$ & $1.68(1.13,2.48)$ & 0.04 \\
\hline \multicolumn{6}{|c|}{ Taurochenodeoxycholic Acid (TCDCA) } \\
\hline N Cases (\%) & $108(19.0)$ & $151(26.5)$ & $142(25.0)$ & $168(29.5)$ & \\
\hline OR $(95 \% \mathrm{Cl})$ & Reference & $1.39(0.98,1.98)$ & $1.37(0.95,1.96)$ & $1.62(1.11,2.36)$ & 0.02 \\
\hline \multicolumn{6}{|c|}{ Glycohyocholic Acid (GHCA) } \\
\hline N Cases (\%) & $152(26.7)$ & $104(18.3)$ & $161(28.3)$ & $152(26.7)$ & \\
\hline OR $(95 \% \mathrm{Cl})$ & Reference & $1.27(0.88,1.82)$ & $1.42(1.00,2.01)$ & $1.65(1.13,2.40)$ & 0.06 \\
\hline
\end{tabular}


Tauro-Alpha, Muricholic Acid (TaMCA)

$\mathrm{N}$ Cases $(\%)$

OR $(95 \% \mathrm{Cl})$

Secondary unconjugated bile acids

Deoxycholic Acid (DCA)

$\mathrm{N}$ Cases (\%)

OR $(95 \% \mathrm{Cl})$

Secondary conjugated bile acids

Glycodeoxycholic Acid (GDCA)

$\mathrm{N}$ Cases (\%)

OR $(95 \% \mathrm{Cl})$

Taurodeoxycholic Acid (TDCA)

$\mathrm{N}$ Cases (\%)

OR $(95 \% \mathrm{Cl})$

Glycolithocholic Acid (GLCA)

$\mathrm{N}$ Cases (\%)

OR $(95 \% \mathrm{Cl})$

Tertiary unconjugated bile acids

Ursodeoxycholic Acid (UDCA)

$\mathrm{N}$ Cases (\%)

OR $(95 \% \mathrm{Cl})$

Tertiary conjugated bile acids

Glycoursodeoxycholic Acid (GUDCA)

$\mathrm{N}$ Cases (\%)

OR $(95 \% \mathrm{Cl})$

Tauroursodeoxycholic Acid (TUDCA)

$\mathrm{N}$ Cases (\%)

OR $(95 \% \mathrm{Cl})$
$201(35.3) \quad 59(10.4) \quad 157(27.6) \quad 152(26.7)$

Reference $\quad 1.15(0.75,1.79) \quad 1.37(0.99,1.91) \quad 1.38(0.98,1.94) \quad 0.09$

$127(22.4) \quad 135(23.8) \quad 159(28.0) \quad 147(25.9)$

Reference $\quad 1.02(0.72,1.45) \quad 1.24(0.86,1.78) \quad 1.11(0.76,1.61) \quad 0.52$

$107(18.8) \quad 145(25.5) \quad 151(26.5) \quad 166(29.2)$

Reference $\quad 1.35(0.94,1.95) \quad 1.38(0.95,2.00) \quad 1.68(1.12,2.54) \quad 0.10$

$113(19.9) \quad 141(24.8) \quad 155(27.2) \quad 160(28.1)$

Reference $\quad 1.25(0.87,1.81) \quad 1.30(0.90,1.87) \quad 1.54(1.02,2.31) \quad 0.08$

$142(25.0) \quad 156(27.4) \quad 138(24.3) \quad 133(23.4)$

Reference $\quad 1.03(0.72,1.46) \quad 0.95(0.66,1.37) \quad 0.91(0.62,1.35) \quad 0.49$

$147(25.8) \quad 140(24.6) \quad 135(23.7) \quad 147(25.8)$

Reference $\quad 0.83(0.58,1.19) \quad 0.89(0.62,1.26) \quad 0.98(0.68,1.41) \quad 0.69$

$130(22.8) \quad 176(30.9) \quad 106(18.6) \quad 157(27.6)$

Reference $\quad 1.36(0.97,1.91) \quad 0.82(0.56,1.19) \quad 1.36(0.94,1.96) \quad 0.36$

$113(19.9) \quad 153(26.9) \quad 157(27.6) \quad 102(17.9)$

Reference $1.12(0.80,1.55) \quad 0.97(0.66,1.44) \quad 1.26(0.88,1.79) \quad 0.24$ 
*Results from conditional logistic regression analyses conditioned on age, sex, study center, follow-up time, time of day at blood collection, fasting status, menopausal status, current use of exogenous hormones, and phase of menstrual cycle, and additionally adjusted for education level, physical activity level, smoking, waist circumference, height, and consumption of red and processed meat, fat, fiber, and energy;

†Two-sided $P$ value for linear trend from logistic regression models with bile acid values on the log2 scale as the independent variable; 


\section{Figure titles and legends}

Figure 1. Odds ratios of colon cancer for individuals in the highest quartile of bile acid concentrations compared to those in the lowest quartile. Results from conditional logistic regression analyses among 569 cases of colon cancer and 569 matched controls, conditioned on age, sex, study center, follow-up time, time of day at blood collection, fasting status, menopausal status, current use of exogenous hormones, and phase of menstrual cycle, and additionally adjusted for education level, physical activity level, smoking, waist circumference, height, and consumption of red and processed meat, fat, fiber, and energy; two-sided P values for linear trend from logistic regression models with bile acid values on the log2 scale as the independent variable; CA (Cholic Acid); CDCA (Chenodeoxycholic Acid); DCA (Deoxycholic Acid); GCA (Glycocholic Acid); GCDCA (Glycochenodeoxycholic Acid); GDCA (Glycodeoxycholic Acid); GHCA (Glycohyocholic Acid); GLCA (Glycolithocholic Acid); GUDCA (Glycoursodeoxycholic Acid); HCA (Hyocholic Acid); TaMCA (Tauro-Alpha-Muricholic Acid); TCA (Taurocholic Acid); TCDCA (Taurochenodeoxycholic Acid); TDCA (Taurodeoxycholic Acid); TUDCA (Tauroursodeoxycholic Acid); UDCA (Ursodeoxycholic Acid);

Figure 2. Odds ratios of colon cancer for individuals in the highest quartile of bile acid concentrations compared to those in the lowest quartile, stratified by sex. Black circles depict odds ratios among women, light circles depict odds ratios among men. Results from 
conditional logistic regression analyses stratified by sex (women: $n$ cases $=356, n$ controls $=$ 356; men: $n$ cases $=213, n$ controls $=213)$, conditioned on age, study center, follow-up time, time of day at blood collection, fasting status, menopausal status (women), current use of exogenous hormones (women), and phase of menstrual cycle (women), and additionally adjusted for education level, physical activity level, smoking, waist circumference, height, and consumption of red and processed meat, fat, fiber, and energy; two-sided P values for linear trend from logistic regression models with bile acid values on the log2 scale as the independent variable; CA (Cholic Acid); CDCA (Chenodeoxycholic Acid); DCA (Deoxycholic Acid); GCA (Glycocholic Acid); GCDCA (Glycochenodeoxycholic Acid); GDCA (Glycodeoxycholic Acid); GHCA (Glycohyocholic Acid); GLCA (Glycolithocholic Acid); GUDCA (Glycoursodeoxycholic Acid); HCA (Hyocholic Acid); TaMCA (Tauro-Alpha-Muricholic Acid); TCA (Taurocholic Acid); TCDCA (Taurochenodeoxycholic Acid); TDCA (Taurodeoxycholic Acid); TUDCA (Tauroursodeoxycholic Acid); UDCA (Ursodeoxycholic Acid); 


\section{Figure 1}

\section{Bile acid}

Primary, unconjugated

Cholic Acid (CA)

Chenodeoxycholic Acid (CDCA)

Hyocholic Acid (HCA)

Primary, conjugated

Glycocholic Acid (GCA)

Taurocholic Acid (TCA)

Glycochenodeoxycholic Acid (GCDCA)

Taurochenodeoxycholic (TCDCA)

Glycohyocholic Acid (GHCA)

Tauro-Alpha-Muricholic Acid (TaMCA)

Secondary, unconjugated

Deoxycholic Acid (DCA)

Secondary, conjugated

Glycodeoxycholic Acid (GDCA)

Taurodeoxycholic Acid (TDCA)

Glycolithocholic Acid (GLCA)

Tertiary, unconjugated

Ursodeoxycholic Acid (UDCA)

Tertiary, conjugated

Glycoursodeoxycholic Acid (GUDCA)

Tauroursodeoxycholic Acid (TUDCA)

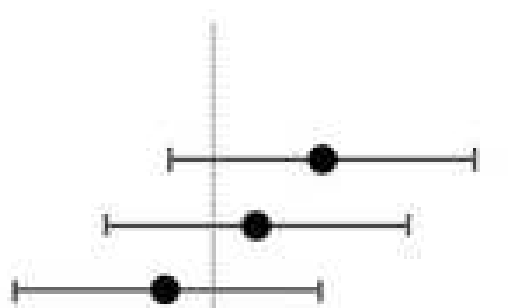

OR (95\% Cl) $\quad p_{\text {trend }}$

$1.32(0.90-1.94) \quad 0.27$

$1.12(0.77-1.64) \quad 0.76$

$0.89(0.61-1.31) \quad 0.60$

$2.22(1.52-3.26) \quad 0.002$

$1.78(1.23-2.58) \quad 0.01$

$1.68(1.13-2.48) \quad 0.04$

$1.62(1.11-2.36) \quad 0.02$

$1.65(1.13-2.40) \quad 0.06$

$1.38(0.98-1.94) \quad 0.09$

$1.11(0.76-1.61) \quad 0.52$

$1.68(1.12-2.54) \quad 0.10$

$1.54(1.02-2.31) \quad 0.08$

$0.91(0.62-1.35) \quad 0.49$

$0.98(0.68-1.41) \quad 0.69$

$1.36(0.94-1.96) \quad 0.36$

$1.26(0.88-1.79) \quad 0.24$ 


\section{Figure 2}

Bile acid

Primary, unconjugated

Cholic Acid (CA)

Chenodeoxycholic Acid (CDCA)

Hyocholic Acid (HCA)

Primary, conjugated

Glycocholic Acid (GCA)

Taurocholic Acid (TCA)

Glycochenodeoxycholic Acid (GCDCA)

Taurochenodeoxycholic (TCDCA)

Glycohyocholic Acid (GHCA)

Tauro-Alpha-Muricholic Acid (TaMCA)

Secondary, unconjugated

Deoxycholic Acid (DCA)

Secondary, conjugated

Glycodeoxycholic Acid (GDCA)

Taurodeoxycholic Acid (TDCA)

Glycolithocholic Acid (GLCA)

Tertiary, unconjugated

Ursodeoxycholic Acid (UDCA)

\section{Tertiary, conjugated}

Glycoursodeoxycholic Acid (GUDCA)

Tauroursodeoxycholic Acid (TUDCA)

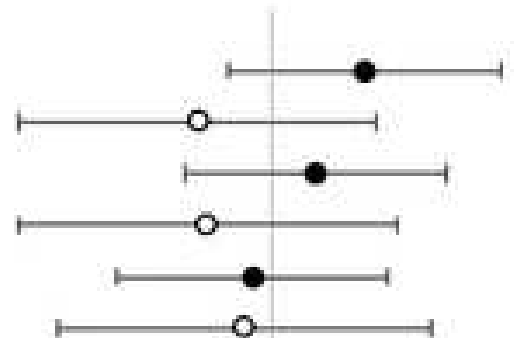

OR $(95 \% \mathrm{Cl}) \quad \mathrm{P}_{\text {taend }}$

$1.41(0.86-2.32) \quad 0.13$

$0.77(0.40-1.47) \quad 0.75$

$1.18(0.73-1.89) \quad 0.34$

$0.79(0.40-1.58) \quad 0.23$

$0.94(0.57-1.53) \quad 0.45$

$0.91(0.46-1.80) \quad 0.84$

$2.52(1.52-4.20)<0.001$

$1.25(0.67-2.34) \quad 0.89$

$2.46(1.49-4,08) \quad 0.006$

$1.18(0.65-2.15) \quad 0.80$

$2.22(1.33-3.71) \quad 0.007$

$0.78(0.40-1.54) \quad 0.68$

$2.23(1.37-3.65\} \quad 0.009$

$0.99(0.53-1.85) \quad 0.99$

$1.71(1.06-2.77) \quad 0.02$

$1.36(0.70-2.65) \quad 0.95$

$1.52(0.88-2.63) \quad 0.46$

$1.65(0.79 \cdot 3.44) \quad 0.46$

$0.91(0.56-1.47) \quad 0.71$

$1.20(0.63-2.31) \quad 0.69$

$1.58(0.92-2.71) \quad 0.19$

$2.20(1.06-4.60) \quad 0.46$

$1.54(0.91-2.62) \quad 0.22$

$1.37(0.69-2.72) \quad 0.44$

$0.76(0.46-1.26) \quad 0.41$

$1.50(0.75-2.99) \quad 0.68$

$0.87(0.54-1.38) \quad 0.65$

$1.00(0.51-1.98) \quad 0.58$

$1.40(0.87-2.25) \quad 0.16$

$1.23(0.61-2.49) \quad 0.41$

$1.33(0.86-2.06) \quad 0.14$

$0.95(0.49-1.83) \quad 0.53$

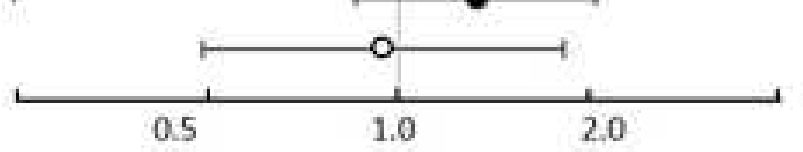

